\title{
LIGHT DIFFRACTION BY FRACTALS: COMPARISON OF EXPERIMENTAL DATA WITH THE OBTAINED BY NUMERICAL METHODS FOURIER IMAGES OF THE OBJECT PICTURES
}

\author{
Galina V. Arzamastseva, Mikhail G. Evtikhov, Feodor V. Lisovsky, Ekaterina G. \\ Mansvetova \\ Kotel `nikov Institute of Radioengineering and Electronics, Fryazino Branch, Russian Academy of Sciences, \\ http:/ / fire.relarn.ru \\ Fryazino, Moscow region 141120, Russian Federation \\ arzamastseva@mail.ru,emg20022002@mail.ru, lisf@df.ru,mansvetova_eg@mail.ru
}

Abstract. An experimental investigation of diffraction of the collimated light beam (with a wavelength of $0.63 \mu \mathrm{m})$ in the zone of Fraunhofer by obtained with computer images of fractals, which are transferred onto a transparent film using an imagesetter with a resolution of 1333 points $/ \mathrm{cm}$ (3386 dpi) and the spot size of $7.5 \mu \mathrm{m}$. Diffraction pattern were visually observed on the screen and recorded using a digital camera and then transferred to the computer for processing. The objects of study were various prefractals of 4th-9th generations: the "classic" (the Sierpinski carpet, a fractal of Vicsek, the Koch snowflake and less known (eg. of L-systems). Observed in experiments diffraction patterns were compared with digitally obtained ones that is, with the Fourier-images of the prefractal pictures, approximated by a mesh function on a uniform square grid at different values of the used in cflculation parameter $p$, determining the ratio between the ratio of the grid period and the smallest prefractal element size. The relation between the values of the parameters $p$ and the degree of compliance of a digital diffraction patterns to the observed experimentally was determined.

Keywords: light difraction, mesh function, fractal, Fourier image, digital method, experiment

UDC 51.74; 535.42

Bibliography - 18 references

Received 25.09.2017

RENSIT, 2017, 9(2):221-229

DOI: $10.17725 /$ rensit.2017.09.221

ConTENTS:

1. INTRODUCTION (221)

2. EXPERIMENTAL CONDitions (222)

3. Procedure for Fourier-images DETERMININATION OF PREFRACTAL PICTURES (222)

4. INVESTIGATION RESULTS DISCUSSION (223)

5. Conclusion (227)

REFERENCES (228)

\section{INTRODUCTION}

Interest in theoretical and experimental study of electromagnetic waves diffraction by flat fractals that emerged at the end of the last century, was not so much due to epistemological reasons, how many opportunities of practical application. In particular, it was shown that the analy-sis of the distribution of the diffracted radiation in the zone of Fraunhofer allows quite easy to define the essential characteristics of fractal objects - Hausdorff dimension [1]. It soon became clear that this does not necessarily actually accomplish quite timeconsuming experiments on the observation of diffraction and the processing of the obtained results, but rather to obtain a graphics image of a fractal (more precisely, prefractal of high enough generation), 
digitize it and continue to use methods of Fourier analysis.

In most cases, to determine the fractal dimension anybody operates in this manner, so the number of works devoted to the study of diffraction itself, is relatively small [2-7]. It is almost not studied the questions remain whether there are differences between real and digital diffraction pattern and what conditions to meet their identity needs to satisfy the procedure for digitizing and processing the pictures of fractals. The aim of this work is to answer these questions.

\section{EXPERIMENTAL CONDITIONS}

A first and necessary step for the implementation of experiments on the observation of diffraction is the creation of black-and-white raster pictures of the selected prefractals on the computer using specially designed software. Then, the resulting pictures were automatically printed onto a transparent film with imagesetter having resolution of 1333 points/cm (3386 dpi) and the spot size of $7.5 \mu \mathrm{m}$. The smallest fractal element was formed from 4-10 points, that is, its linear size was in the range of 30 to 75 microns. The described method of picture forming made it possible to obtain very high contrast, almost unattainable for traditional photography.

In Fig. 1 the block diagram of experimental setup used for observation of the diffraction pattern forming in the Fraunhofer zone after the passage of the light beam through the transparent film with prefractal picture on its surface

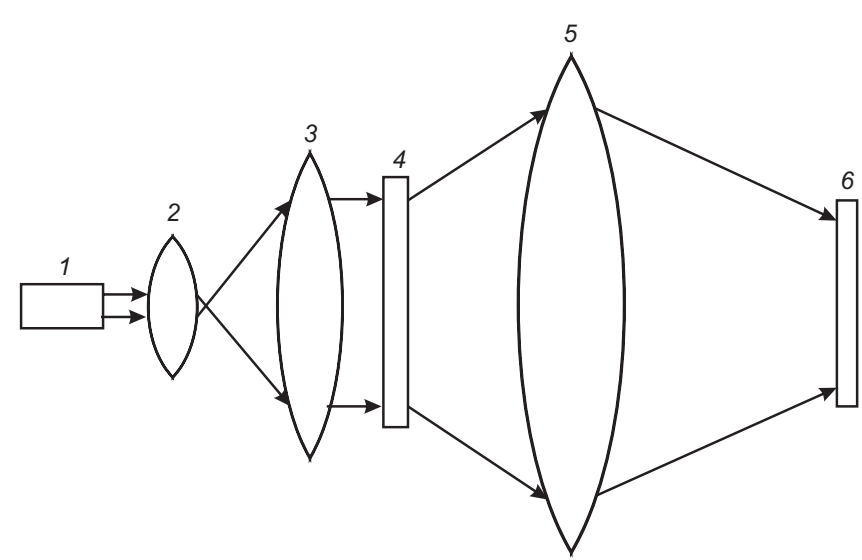

Fig. 1. A block diagram of the optical setup for observing diffraction pattern in the Fraunhofer zone.

is shown. A narrow beam of light with a wavelength of 0.63 microns from a helium-neon laser 1 is expanded and collimated using a system of confocal lenses 2 and 3 to diameter 5 to $8 \mathrm{~cm}$ and then directed onto the transparent film 4 with the picture of a fractal object. The lens 5 forms an image of the diffraction pattern in the plane of diffraction, where the screen 6 is mounted. Instead of screen one can use a computer coupled with a digital camera and subject the observed diffraction pattern to the necessary treatment.

\section{PROCEDURE FOR FOURIER- IMAGES DETERMININATION OF PREFRACTAL PICTURES}

Created with help of computer blackand-white raster pictures of prefractals were approximated by a grid function on a uniform grid with number of nodes $n_{1} \times n_{2}$, where the values $n_{1}$ and $n_{2}$ were chosen sufficiently large (up to 4096) in order to ensure adequate approximation of the smallest details and to have the opportunity to explore generation of prefractals with high numbers of generations. For thus digitized pictures using fast Fourier 
transform one can determine the values of the Fourier component squared modules, i.e. the spectral distribution of intensity $I$ of diffracted radiation in the zone of Fraunhofer. To display the intensity Iof the diffraction maxima on the $2 D$-plane, one can use representation of $I$ values in the form of circles with radius proportional to intensity (or logarithm of it), where coefficient of proportionality is chosen for reasons of optimal demonstrativeness of images; to reach that additional Gaussian blur of circles was also used.

Used in the present work a procedure for determination of fractal object picture Fourier-images (i.e., "digital diffractograms"), previously described and applied to study the fractal-like domain structures in magnetic films [8], later was applied to the real test objects (mono- and bi-periodical domain structures), when it was possible in the visible range of wavelengths to observe directly and photograph the diffraction pattern by the "transmission" mode [9]. Afterwards similar numerical methods have been used for many other fractal objects [10-15].

For all the studied geometric fractals on diffraction patterns, experimentally obtained by optical method, and digital diffractograms there is a difference between central and peripheral parts. Localized near the center portion has a radial-ring structure of the location of diffraction maxima and has the self-similarity inherent for any fractal objects, while the having no self-similarity peripheral part characterized by an equidistant arrangement of diffraction maxima along the defined number of radial directions (rays). For the first time this fact drew the attention of the authors of article [2] devoted to the study of optical Fraunhofer diffraction on the classic Koch snowflake, where they are to refer to the central and peripheral parts used the terms "fractal part" and "lattice part" and linked the appearance of the latter with the fact that in a twodimensional set of elements forming the fractal, one can select a one-dimensional diffraction gratings consisting of equally oriented elements (eg. segments). Later this mechanism was discussed in detail on the example of the family of the generalized process of the Koch fractals $[14,15]$.

Although in all the above cases it was possible to speak about the good correspondence between the experimentally observed and digital diffractograms, it is almost not studied the questions remain whether there are differences between real and digital diffraction pattern and what conditions to meet their identity needs to satisfy the procedure for digitizing and processing the pictures of fractals. The aim of this work is to answer these questions.

\section{INVESTIGATION RESULTS AND DISCUSSION}

The main attention was paid to studying the influence of the parameter $p$ equal to the ratio of the dimensional size of the smallest element of prefractal to the period of the grid, on the degree of compliance of a digital diffractograms to the experimentally observed diffractional 
patterns at different values of $p$ which in the calculations was varied by changing the period of the grid for the same computer-generated raster image of the selected prefractal. If the value of $p$ is close to one, only fractal part of the real diffraction patterns is displayed on the digital diffractogram; with increasing of $p$ the lattice part appears, which with the growth of $p$ monotonically approaches to the existing in the real diffraction pattern. We studied the fractals of two types, in which the smallest element ("seed") is either a square or a line segment.

Let's start the presentation with two described in detail in [11] fractals $L$-systems with the "seed" in the form of a square [16-18]; then - fractals LS1 and LS2. Algorithms of construction of these fractals can be represented using the following recurrence relations, showing successive transformation of a single seed of a square on the complex plane [11]. Note that these algorithms contain a transform that uses only integer translation of the object along the real and (or) the imaginary axis, and turns only at angles that are multiples of $\pi / 2$.

If one selects the orientation of the coordinate system on the complex plane so that the imaginary axis was directed horizontally to the right and the real axis is horizontal down, and place a single seed box (set $Z^{(0)}$ ) in the first quadrant (the coordinates of the vertices are $(0,0),(0, i)$ $(1, \mathrm{i})$, and $(1,0))$, then the LS1 prefractal any order (generation) $Z^{(\text {n) }}$ obtained by operation of merging sets $U$ with the help of recursion relations [11]:
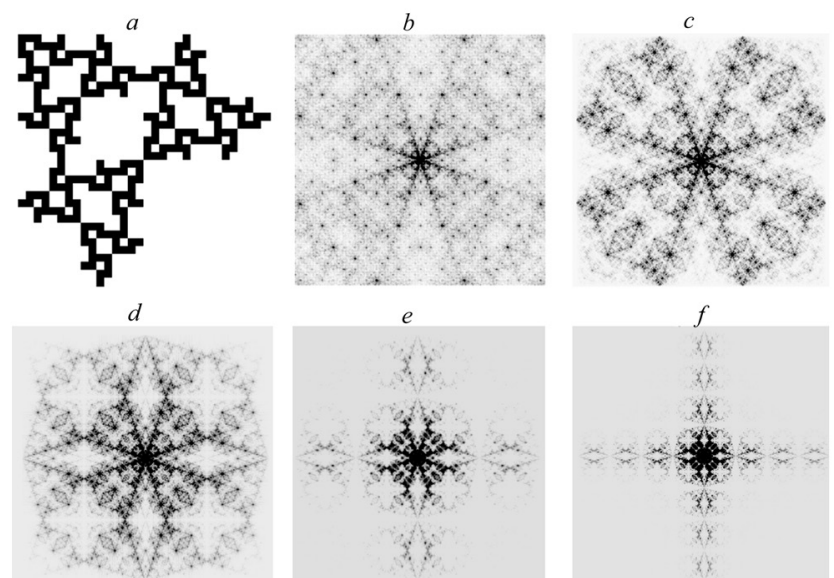

Fig. 2. Prefractal LS1 of 4th-generation picture (a) and digital diffractograms for prefractal of 7 th generation with values of $p$ equal to $0.5(b), 1.0(c), 2.0,(d), 4.0(e)$ and $8.0(f)$.

$Z^{(\mathrm{n}+1)}=Z^{(\mathrm{n})} \bigcup\left(i Z^{(\mathrm{n})}+(1+i) 2^{n}\right) \bigcup\left(-Z^{(\mathrm{n})}+(1+i) 2^{\mathrm{n}}\right)$.

For a fractal LS2 chain of consecutive transformations for set in a form of a single square seed is given by the expressions [11]

$Z^{(\mathrm{n}+1)}=Z^{(\mathrm{n})} \cup\left(-Z^{(\mathrm{n})}+(2+i) 2^{n}\right) \cup\left(-Z^{(\mathrm{n})}+(1+2 i)^{\mathrm{n}}\right)$.

Fig. 2a shows an image of prefractal LS1 of the 4th generation; digital diffractogram for prefractal of 7 th generation with the parameter $p$ values equal to $0.5,1.0,2.0,4.0$ and 8.0 are shown in Fig. 2b-2f, respectively. It is seen that the structure of the digital diffraction patterns is very strongly dependent on the parameter $p$, especially at small values of it (see Fig. 2b), when there is a stark contrast to the experimentally obtained for prefractal of the same generation diffraction pattern, shown in Fig. 3. Nevertheless, even at $p=1$ (the cell size of the grid functions is equal to the size of the smallest element of prefractal), the digital diffraction pattern (Fig. 2c) clearly displays fractal part, although lattice part is completely absent This result does not depend on the prefractal generation 


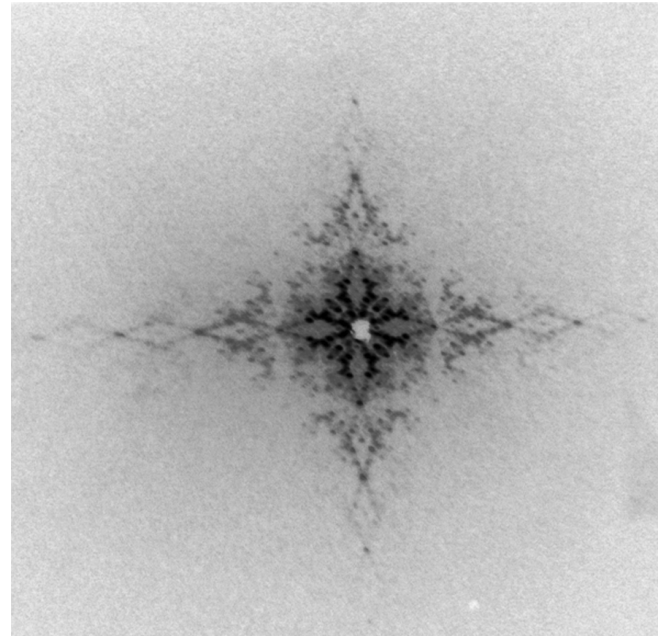

Fig. 3. Experimental diffraction pattern for prefractal LS1 of 4th-generation.

number; but for high generations details of the fractal part of the digital diffractogram manifest more clearly.

At $p \geq 2$ on digital diffractogram appears lattice part, which increases with a further decrease of cell size of the grid functions with respect to the size of the smallest element of prefractal (Fig. 2df). Comparison digital diffraction pattern
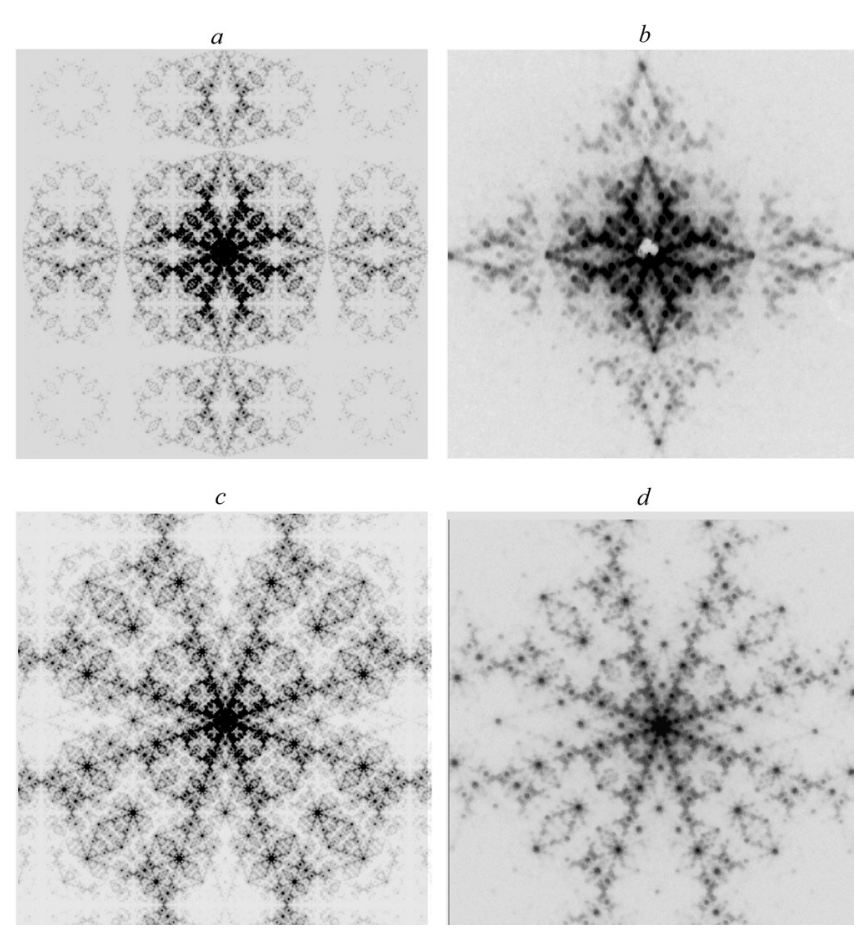

Fig. 4. Close-up (in two different scales) pictures of central part of the digital $(a, c)$ and experi-mental $(b, d)$ diffraction patterns from prefractal LS1 of 7th-generation.
(Fig. 2f) with the experimental one (Fig. 3) shows that they coincide with a high degree of accuracy. About this testify and close-up (in two different scales) central part pictures of digital and experimental diffraction patterns, which are given next in Fig. 4a,c and Fig. 4b,d.

Similar results were obtained for a fractal LS2, the picture of which for prefractal of 4-th generation are shown in Fig. 5a and digital diffractogram for prefractal of 9-th generation at $p=1$ - in Fig. 5b and at $p=4-$ in Fig. 5c. Experimental diffraction pattern for

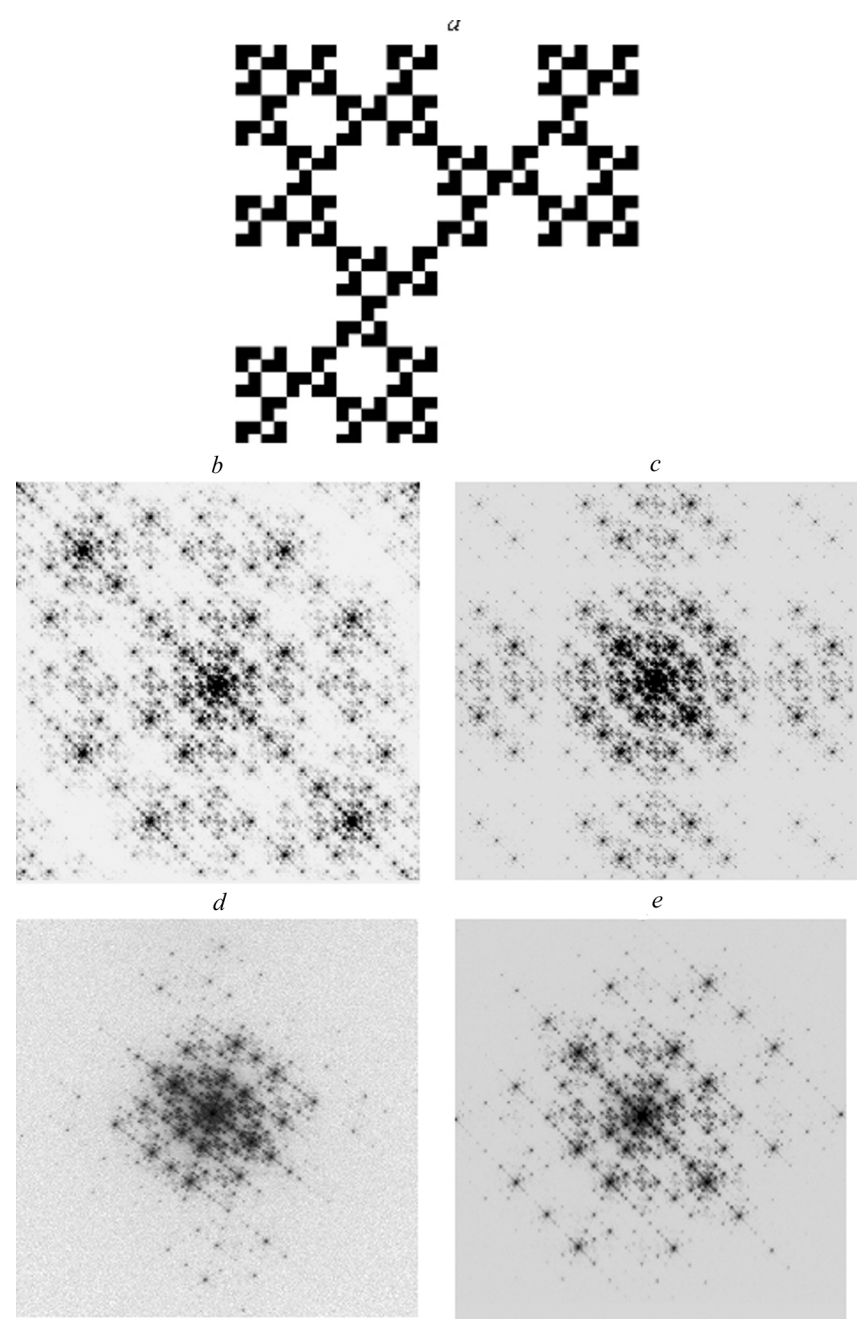

Fig. 5. Picture of prefractal LS2 of 4th-generation (a), digital difractograms for prefractal of 9 th generation with values of equal to 1.0 (b) and 4.0 (c), and picture of the experimental difractogram for prefractal of 9th generation in two different scales (d-general appearance, $e-$ close-up central part). 
prefractal of 9-th generation is shown in two different scales in Fig. 5d (general appearance) and Fig. 5e (close-up) are well consistent with the calculated at $p=4$.

The comparison of experimental and digital diffraction patterns was conducted and for other objects: Vicsek fractal, Sierpinski carpet and Koch snowflake. Fig. 6a shows the image of 3rd generation Vicsek prefractal, and Fig. $6 \mathrm{~b}$ and Fig. $6 \mathrm{c}-$ the digital diffractograms of 5 th generation prefractal for $p=1$ and for $p=8$.
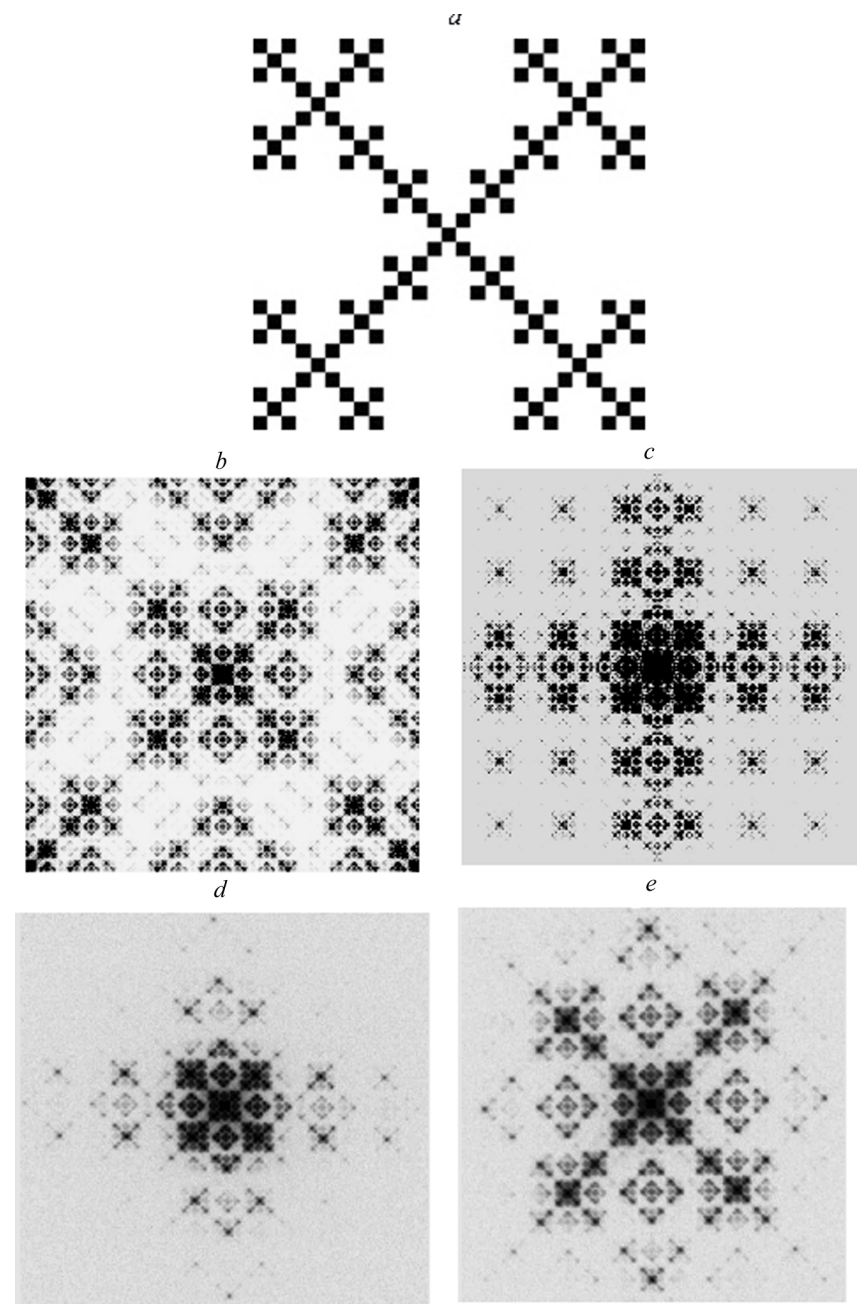

Fig. 6. Picture of Vicsek prefractal of 3rd-generation (a), digital diffractograms for prefractal of 4 th generation for $p$ $=1$ (b) and $p=9$ (c), and picture of the experimental difractogram for prefractal of 4 th generation in two different scales (d - general appearance, e - close-up central part).
Experimental diffraction patterns for prefractal of 5 -th generation shown in two different scales in Fig. 6d (general appearance) and Fig. 6e (close-up) are in good agreement with the calculated for $p=8$. Sierpinski carpet of the $3 \mathrm{rd}$ generation is shown in Fig. 7a, and Fig. $7 \mathrm{~b}$ and Fig. $7 \mathrm{c}$ presents the calculated diffraction pattern for this prefractal of 4th generation with $p=1$ and $p=9$, respectively. Experimental difractogram for prefractal of the same generation at two different scales is shown in Fig. $7 d$
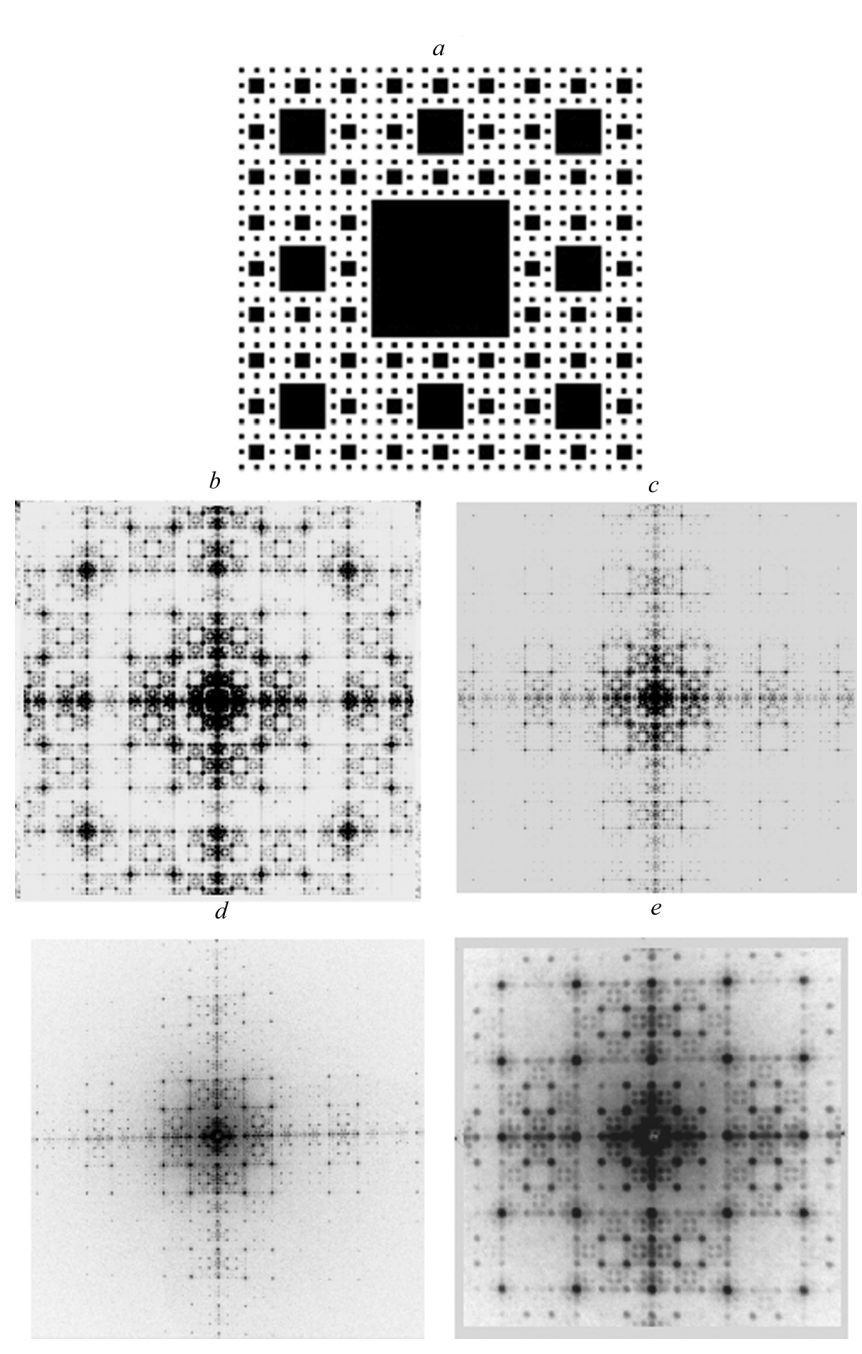

Fig. 7. Picture of Sierpinski carpet of the 3rd generation (a), digital diffractogram for prefractal of 5 th generation for $p=1$ (b) and $p=4$ (c), and picture of the experimental difractogram for prefractal of 5 th generation in two different scales (d-general appearance, $e-$ close-up central part). 
(general appearance) and Fig. 7e (closeup central part). Experimental and
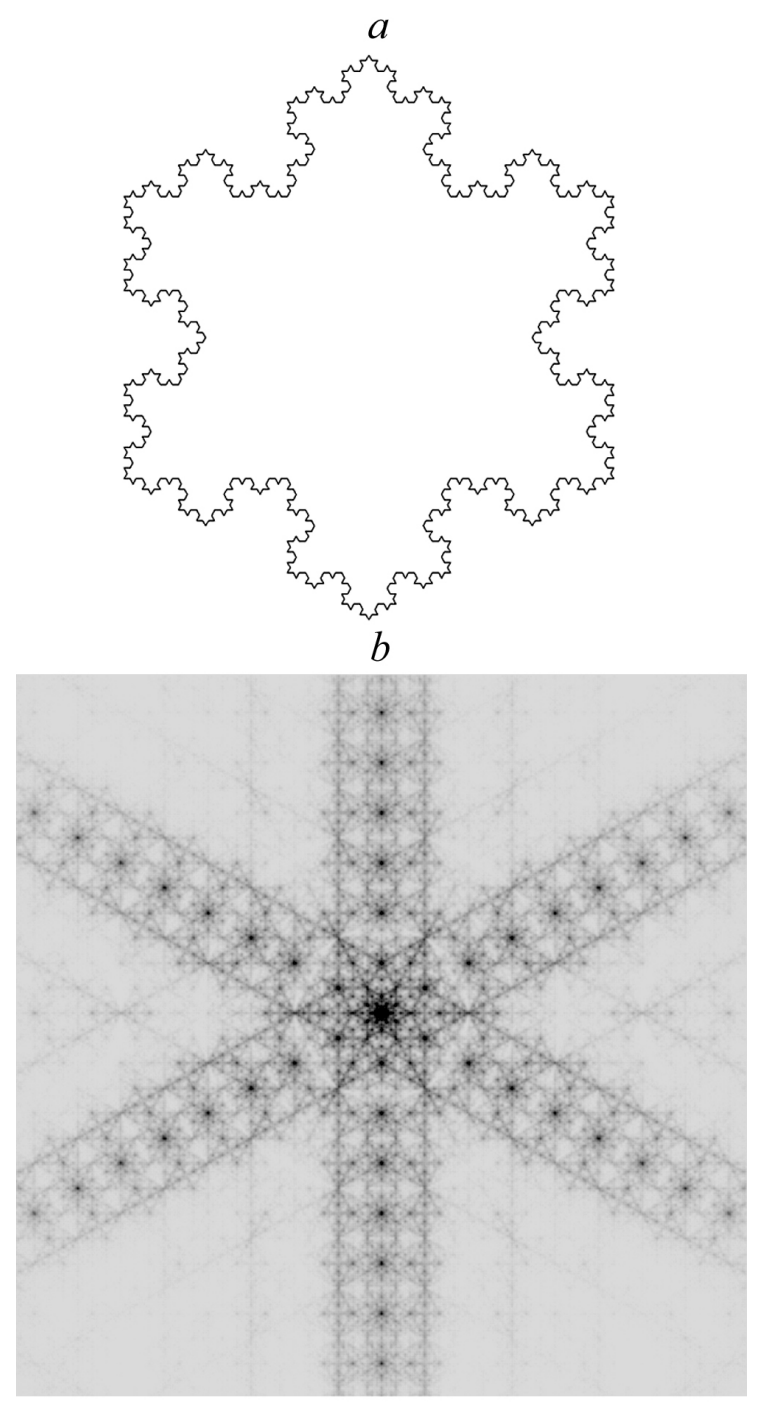

c

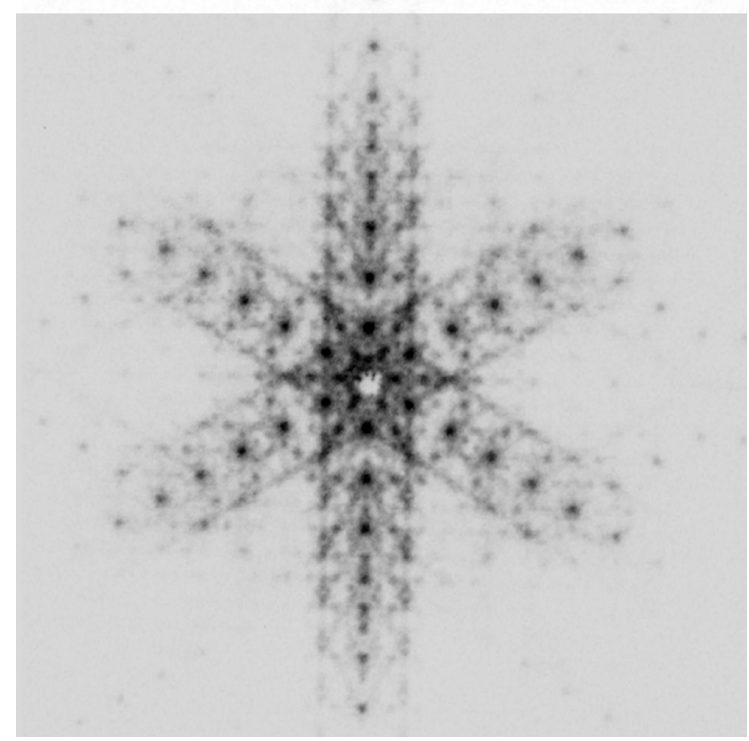

Fig. 8. Koch snowflake of the 4th generation picture (a) and digital (для р (b)) and experimental (c) diffraction patterns. digital diffractograms for 5 th generation Vicsek prefractal with $p=8$ and for 4 th generation Sierpinski carpet with $p=9$ fully correspond to each other.

The Koch snowflake is fundamentally different from the discussed above fractals, the fact that the element of minimal size in it is not a square, but a segment of line which cannot be displayed by one cell of the grid functions and to adequate representation it in calculations, one needs more cells. Therefore, in consideration of diffraction by the Koch snowflake by using the fast Fourier transform it should be automatically established the condition $p \geq 2$, under which the digital diffraction pattern is in good agreement with experimental one and lattice part is present. Almost coincident with each other digital (at $p=20)$ and experimental diffraction patterns for represented in Fig. 8a the 4-th generation Koch snowflake, are shown in Fig. $8 \mathrm{~b}$ and Fig. 8c, respectively.

\section{CONCLUSION}

Analysis of all the obtained experimental and digital diffraction patterns showed that with increasing generation number of prefractal per unit leads to enlarging of fractal part by $m$ times, where $m$ is the scale factor. Additional confirmation of this conclusion was obtained in [14], where was obtained the digital diffractogram for the generalized Koch curve with an arbitrary apex angle $\alpha$, scale factor of which varied from 2 (when $\alpha \rightarrow 0^{\circ}$ ) to 4 (when $\alpha \rightarrow 180^{\circ}$ ). For fractals LS1 and $L S 2$, the scaling factor is 2 , and for a fractal 
of Vicsek, Sierpinski carpet, and classic Koch snowflake equals 3. The result for the Koch snowflake is the same as that found in [2], the authors relied not only on the results of the experiments, but also on rigorous analytical calculations. In the limit, as the generation number of prefractal leads to infinity, the fractal part should completely displace the lattice.

The results of investigations of diffraction on different fractal objects show that if the condition $p \geq 2$ is met when the size of the smallest element of prefractal more than two times the period of the grid functions obtained by fast Fourier transform digital diffraction pattern are in good agreement with the experimental ones. Received any way diffractograms have not only fractal, but also the lattice part, which arises from the fact that the generation number of prefractal always bounded above. When $p<1$ the digital pattern is far from experimentally observed, and for values $1 \leq p<2$ digital methods correctly reflect only the central (fractal) part.

\section{REFERENCES}

1. Alain C, Cloitre. M. Phys. Rev. B, 1986, 33(5): 3566-3569.

2. Uozumi J, Kimura H, Asakura T. J. Mod. Optics, 1990, 37(6): 1011-1031.

3. Sakurada Y, Uozumi J, Asakura T. Opt. rev., 1994, 1(1): 3-7.

4. Uozumi J, Kimura H, Asakura T. J. Mod. Optics, 1991, 38(7):1335-1347.

5. Sakurada Y, Uozumi J, Asakura T. J. Optics. A: Pure Appl.Optics, 1992, 1: 29-40.
6. Chabassier G, Angéli B, Heliodore F, Le Mehauté A. J. Optics. A: Pure Appl. Optics, 1992, 1: 41-54.

7. Bo Hou, Gu Xu, Wen W, Wong GK L. Appl. Phys. Lett., 2004, 85(25): 6125-6127.

8. Arzamastseva GV, Evtikhov MG, Lisovsky FV, Lukashenko LI. Trudy XIX Mezhdunarodnoi shkoly-seminara "Novye magnitnye materialy microelectroniki", Moscow, 2004, p. 632-634 (in Russ.).

9. Arzamastseva GV, Evtikhov MG, Lisovsky FV, Mansvetova EG, Temiryazeva MP. ZhETP, 2008, 134(2): 282-290. (in Russ.).

10. Arzamastseva GV, Evtikhov MG, Lisovsky FV, Mansvetova EG. Iqv. RAS, ser. fir., 2010, 74(10):1430-1432 (in Russ.).

11.Arzamastseva GV., Evtikhov MG, Lisovsky FV, Mansvetova EG. Radioelektronika. Nanosistemy. Informatsionnye Tekbnologii (RENSIT), 2012, 4(2): 93-107 (in Russ.).

12. Arzamastseva GV., Evtikhov MG, Lisovsky FV, Mansvetova EG. Electromagnitnye volny $i$ electronnye sistemy, 2012, 17(7): 29-32 (in Russ.).

13. Arzamastseva GV., Evtikhov MG, Lisovsky FV, Mansvetova EG. Electromagnitnye volny $i$ electronnye sistemy, 2012, 17(7): $48-58$ (in Russ.).

14. Arzamastseva GV., Evtikhov MG, Lisovsky FV, Mansvetova EG. RENSIT, 2016, 8(1): 81-90 (in Russ.).

15. Arzamastseva GV., Evtikhov MG, Lisovsky FV, Mansvetova EG. RENSIT, 2016, 8(2): 207-214 (in Russ.). 
16.Lindermayer A. J. Theor. Biol., 1968, 18(3):280-299.

17.Lindermayer A. J. Theor. Biol., 1968, 18(3):300-315.

18.http://lcni.uoregon.edu/ dow /

Geek_art/Binary_fractals/Binary_ fractal_images.html. 